\title{
Anabases
}

ANABASES Traditions et réceptions de l'Antiquité

$21 \mid 2015$

Varia

\section{Lin FOXHALL, Studying Gender in Classical Antiquity}

\section{Geneviève Hoffmann}

(2) OpenEdition

Journals

Édition électronique

URL : http://journals.openedition.org/anabases/5348

DOI : 10.4000/anabases.5348

ISSN : 2256-9421

Éditeur

E.R.A.S.M.E.

Édition imprimée

Date de publication : 1 avril 2015

Pagination : 272-274

ISSN : 1774-4296

\section{Référence électronique}

Geneviève Hoffmann, « Lin foxHALL, Studying Gender in Classical Antiquity », Anabases [En ligne], 21 | 2015, mis en ligne le 01 avril 2015, consulté le 22 septembre 2020. URL : http://

journals.openedition.org/anabases/5348; DOI : https://doi.org/10.4000/anabases.5348

Ce document a été généré automatiquement le 22 septembre 2020.

(c) Anabases 


\title{
Lin FOXHALL, Studying Gender in Classical Antiquity
}

\author{
Geneviève Hoffmann
}

\section{RÉFÉRENCE}

Lin FOXHALL, Studying Gender in Classical Antiquity, Cambridge, Cambridge University

Press, 2013, $188 \mathrm{p}$.

19, 99 livres/ ISBN 978-0-521-55739-9

1 Lin Foxhall (LF), professeure à l'université de Leicester, archéologue et spécialiste de l'agriculture grecque, s'intéresse également à la question du genre. Elle a publié deux livres avec John Salmon sur la masculinité dans l'Antiquité (1998). Le présent ouvrage, paru dans la collection Key Themes in Ancient History des Presses de Cambridge, est un manuel destiné plus particulièrement aux étudiants engagés dans les études classiques afin de les initier à cet outil d'analyse qu'est le genre. Six domaines ont été retenus, chaque chapitre étant divisé en rubriques centrées sur le commentaire d'un document, d'un texte, d'une découverte archéologique ou d'une représentation iconographique. Ainsi, des sources antiques bien connues sont-elles réexaminées à la lumière des problématiques que soulève la construction du genre.

2 Le premier chapitre " Gender and the study of classical antiquity ", tient lieu à la fois d'introduction, d'historiographie de la question et de présentation des sujets traités (p. 1-23). Dans le sillage des réflexions théoriques de Judith Butler, l'auteure souligne la pertinence du genre comme critère épistémologique pour remettre en cause le clivage « naturel » entre féminin et masculin. Elle retrace l'histoire de la notion qui, née du féminisme, a fini par inclure les " gays ». Pour souligner le point de vue traditionnel, elle retient le livre de Jérôme Carcopino La Vie quotidienne à Rome à l'apogée de l'Empire (1939) qui resta influent dans les études universitaires jusqu'aux années 1980 du siècle dernier. Dans cet ouvrage, l'historien reprenait sans critique le point de vue moral des auteurs latins qui peignent le portrait de la matrone romaine idéale, à la fois épouse 
irréprochable et excellente mère. Soumis aux élites des mâles dominants du passé comme du présent, les historiens n'étaient pas plus à l'aise pour étudier la question féminine que l'homosexualité. L'auteur pose les jalons les plus importants dans la prise de conscience féministe en France, au Royaume-Uni et aux États-Unis, qui ont permis de sortir de cet obscurantisme. En France, elle rappelle le rôle des Annales, l'importance du Deuxième Sexe de Simone de Beauvoir (1949) et de l'Histoire de la sexualité de Michel Foucault (1976). Les Anglo-Saxons ont découvert la place des femmes dans l'Antiquité dans les années 1970 et 1980. La revue américaine Arethusa publia en 1973 un numéro consacré à cette question dans une perspective féministe. Le livre de Sarah Pomeroy Goddesses, Whores, Wives and Slaves : Women in Classical Antiquity (1975), fut en son temps révolutionnaire. L'auteure dresse un tableau des articles publiés sur les femmes et le genre dans trois périodiques, American Journal of Philology (USA), Classical Quarterly (Grande-Bretagne) et Historia (Allemagne) (p. 9). Cette présentation historiographique éclaire de façon didactique les conditions d'émergence du genre, dans une perspective anglo-saxonne. Elle entend convaincre les historiens de l'intérêt d'interroger une documentation bien connue par cette nouvelle catégorie de pensée.

Le chapitre 2 (p. 24-44) qui traite du domaine et de la famille commence par l'étude du vocabulaire grec (oikos, genos, angchisteia) et latin (familia, paterfamilias, domus), puis présente les idées d'Aristote et de Cicéron sur le sujet. Postulant que la " gender hierarchy " est au cœur de l'organisation des sociétés antiques (p. 30), LF étudie sous cet angle le mariage grec et le mariage romain avant d'autopsier le couple par le biais de la relation adultérine. La conclusion est attendue : dans le couple, les hommes ont le pouvoir et les femmes sont soumises. Le chapitre 3 porte sur la démographie (p. 45-67). Sous ce titre, il ne faut pas entendre des données chiffrées, mais une réflexion sur la généalogie, sur l'exposition et l'éducation des enfants, sur la puberté, la mort et le culte de la mémoire. La conclusion le souligne : dès l'enfance, garçons et filles sont perçus et traités différemment par la famille qui est le lieu où les rôles se cristallisent pour les conduire sur des voies différentes (p. 67). La question du corps s'ouvre dans le chapitre 4 (p. 68-89) sur le cas particulier de l'eunuque Favorinus qui se présentait comme doté des deux natures, féminine et masculine. L'étude de la reproduction permet de reprendre les termes du débat sur le rôle respectif des parents dans la conception, d'Eschyle à Aristote. Puis sont brièvement traités et sans souci de grande cohérence le point du vue hippocratique sur le corps, le portrait romain, le comportement sexuel et le corps politique à Athènes, la violence dans la guerre... La conclusion impose l'idée que les corps sont construits par les pratiques et les habitudes sociales, en laissant peu de marge de liberté. L'auteure écrit : « Generally to be a (free, adult and usually elite) man was to have (or acquire) the body of a man " (p. 89), tout en reconnaissant des comportements déviants chez les Grecs comme chez les Romains. Pour en témoigner, LF revient sur l'exemple de l'orateur Favorinus qui en dépit de sa marginalité sexuelle, a joui d'un statut élevé et d'une reconnaissance sociale. Dans le chapitre 5 (p. 91-113), les exemples retenus entendent démontrer que les femmes accédaient difficilement à la richesse et qu'à Rome et à Athènes, elles avaient des activités "étonnamment semblables" (p.98). Elles s'occupaient des enfants, elles étaient chargées de la préparation des repas et de la confection des vêtements, et elles étaient des "sex workers ". C'est incontestable : l'idéologie du genre, qui pesait sur la répartition des travaux et des métiers, entravait l'accès des femmes au pouvoir économique. Le chapitre 6 sur les espaces privés et publics reconnaît la difficulté de faire correspondre les sources littéraires aux testimonia archéologiques et en particulier, d'identifier un 
gynécée dans les maisons grecques. Peu d'espaces sont en effet " gendered in absolute sense ", comme l'est le gymnase (p.114-136). Dans le chapitre 7 qui porte sur les rapports entre religion et société (p. 137-157), sont analysés successivement le sacrifice, les Grandes Panathénées, les Thesmophories à Athènes, les cultes civiques de la Bona Dea et les cultes de Bacchus à Rome, les dédicaces votives et enfin la magie. Pour l'auteure, si les activités religieuses donnaient aux femmes un rôle important, elles renforçaient leur marginalité dans le quotidien.

En conclusion (chapitre 8, p. 158-159), LF reconnaît la difficulté qu'il y a à maîtriser un sujet d'une telle ampleur dans un manuel de 188 pages. Comme elle n'a pu développer certaines problématiques, elle a complété chaque chapitre par une bibliographie commentée. L'intérêt de l'ouvrage réside dans la volonté affichée, mais pas toujours suivie d'effet, de ne pas réduire toutes les problématiques aux rapports de sexe. La tentation est grande en effet de vouloir tout expliquer par la loi du genre, ce " persistent and pervasive subject ", mais pour qu'il soit pertinent, cet outil d'analyse doit être combiné avec d'autres critères : l'âge, la classe, la richesse, autant d'axes de recherche que ce livre ne peut qu'évoquer. On regrette le manque de chronologie et de mise en perspective des sources. Les fiches présentées sont liées les unes aux autres sans cohérence ni souci de transition. Ainsi sont mises sur le même plan, la Sparte de Plutarque, l'Athènes de Périclès, la cité de Gortyne... Comme on trouve ce que l'on cherche, les exemples retenus dégagent une norme qui illustre le postulat du genre. À lire ce manuel, on ne peut pas comprendre les destins de femmes d'exception comme Artémise d'Halicarnasse, Aspasie ou Olympias. Les étudiants francophones ne trouveront dans la bibliographie générale aucun des livres en langue française qui ont renouvelé récemment ce sujet (p. 165-184). Pour s'initier au genre, ils auront tout intérêt à lire Hommes et femmes dans l'Antiquité. Le genre : méthodes et documents, ouvrage publié sous la direction de Sandra Boehringer et Violaine Sébillotte-Cuchet (2011), dont l'approche didactique respecte la spécificité des sources tout en proposant de fructueuses pistes de recherche.

\section{AUTEUR \\ GENEVIÈVE HOFFMANN \\ Université de Picardie Jules Verne genevieve.hoffmann@wanadoo.fr}

